\title{
Cardiac Arrest and Seizure Following Cannabis Overdose and Alcohol Withdrawal
}

\author{
Ashfaq Hussain, FCAl'; Pradipta Bhakta, MD, DNB, MNAMS, FCAI, EDRA ${ }^{2 *}$; \\ Vikash Singh, MD, FCAI, EDRA3 ; Jubil Thomas, MD, FCARCSI, FJFICMI, CCST, HCM'; Edyta Zietak, BSc ${ }^{4}$
}

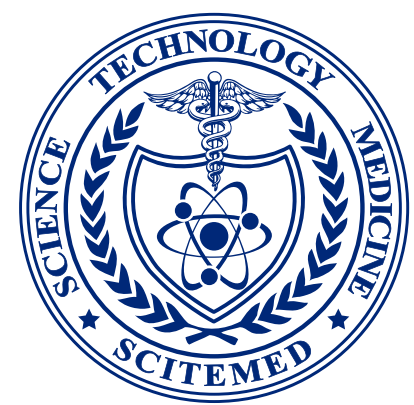

\author{
${ }^{1}$ Department of Anaesthesia and Intensive Care, Our Lady of Lourdes Hospital, Drogheda, Ireland \\ 2 Department of Anaesthesia and Intensive Care, University Hospital Limerick, Dooradoyle, Limerick, Ireland \\ ${ }^{3}$ Department of Anaesthesia and Intensive Care, Tallaght Hospital, Dublin, Ireland \\ ${ }^{4}$ University of Management and Administration, WisZia, Zamosc, Poland
}

\begin{abstract}
Cannabis remains one of the most universally abused recreational drugs. Cannabis abuse can have several cardiovascular adverse effects and an overdose can lead to cardiac arrest. Binge alcohol drinking and subsequent withdrawal in the background of cannabis overdose can lead to convulsion and QT prolongation leading to malignant arrhythmia. We report two cases of ventricular fibrillation complicated with seizure precipitated by cannabis overdose and alcohol withdrawal. Such serious clinical presentation complicated with cardiac arrest does not necessarily lead to a fatal outcome if timely medical attention and care are provided. Timely aggressive resuscitative measures combined with intensive care can lead to a successful outcome. Later on, psychiatric counseling and occupational and behavioral therapies can help them to live longer.
\end{abstract}

\section{Introduction}

Cannabis remains one of the most universally abused recreational drugs worldwide and its popularity has increased considerably due to the recent legalization and medicinal use [1-5]. Sometimes, cannabis overuse can lead to malignant arrhythmia leading to cardiac arrest [5,6]. Alcohol withdrawal following binge drinking combined with cannabis abuse can lead to malignant arrhythmia, seizure, and at times culminating in cardiac arrest $[2,5,6]$. We report two cases of ventricular fibrillation (VF) complicated with seizure caused by cannabis overdose and alcohol withdrawal following binge drinking.

\section{Case Report}

\section{Case 1}

A 22-year-old female was found collapsed at home where she received cardiopulmonary resuscitation (CPR) on the spot. In the ambulance, she had witnessed seizure and her breathing became erratic. She was defibrillated for having VF. On arrival to our emergency department, her Glasgow Coma Scale (GCS) was 3/15 and she was intubated. Her vital parameters were stable, but she was acidotic (Table 1). Her urine was found to be positive for cannabis (quantitative estimation was not available). Her computerized tomography (CT) scan of brain and lumbar puncture report were found to be normal. She was transferred to our intensive care unit (ICU) for subsequent care.

When inquired, her relatives told that she smoked several cannabis cigarettes (amounts unknown) and had several units of wine (1 unit of wine in Ireland amounts to ten gram of alcohol) previous night in a party. She was a diagnosed case of asthma and epilepsy for last three years, for which she was currently not in any treatment. She was clinically seizure-free for the last two years. She was a regular cannabis abuser.

In the ICU, she was kept sedated and received phenytoin and levetiracetam for seizure control. She was found to have prolonged rate-corrected QT interval on her electrocardiogram (ECG), for which she received intravenous magnesium sulphate. Subsequently, she was weaned from the ventilator and was extubated after four days. Later, she was discharged to the ward from where she went home.

\section{Case 2}

A 24-year-old female was brought to our emergency department after she was found collapsed at home. She smoked several cannabis cigarettes and had several units of wine previous night (again the exact amount was not known). She received CPR and was shocked once for having VF. She was found to be unconscious (GCS of 3/15) and thus was intubated. She had a history of deep venous thrombosis in 2012 and was a regular street drugs abuser (clear history not revealed to us). Her urine was found to be positive for benzodiazepine and cannabis (again the quantitative test was not available). Her CT scan of the brain was found to be normal. She was later transferred to our ICU for subsequent management. She developed recurrent episodes of seizure even after treatment with intravenous phenytoin and midazolam. Her seizure could be controlled with intravenous infusion of phenytoin and regular levetiracetam. She was also found to have prolonged rate-corrected QT interval, for which she received intravenous magnesium as she had a malignant arrhythmia. Finally, she was extubated and discharged to home after 8 days.

Both these patients were kept under psychiatry follow-up and evaluation. To the best of our knowledge, neither of them had any similar event.

\section{Discussion}

Majority of effects of cannabis, including serious cardiac side effects, are mediated by active ingredient delta-9-Tetrahydrocannabinol, a strong myocardial stimulant, through cannabis receptors [1-3]. Cannabis has been reported to cause several types of arrhythmias due to direct effect as well as sympathetic stimulation [1]. It is also known to be a proconvulsant or an anticonvulsant agent [3].

Binge drinking has also been reported to be proarrhythmogenic $[1,4]$. Alcohol withdrawal can also lead to prolonging rate-corrected QT interval precipitating in malignant arrhythmias [4,5]. Adding to this, the alcohol withdrawal can precipitate seizure typically manifesting after 6-48 hours $[3,6]$.

Cannabis in combination with alcohol produces pronounced synergetic myocardial effect culminating in life-threatening VF complicated 
with seizure, which can lead to cardiac arrest [1,3]. Both the patients consumed a high amount of cannabis and alcohol (although the exact figure was not known) and were chronic abusers of both. Also, to admit that, this VF combined with seizure might have been caused by cannabis overdose or alcohol withdrawal. It was not possible for us to pinpoint the exact etiology, as both factors were well known to cause such adverse effects.

These two case reports showed that the combined massive ingestion of alcohol and cannabis and the induced cardiac arrhythmia could lead to a serious cardiac event propagating to cardiac arrest. Cannabis is known to induce myocardial ischemia and malignant arrhythmia in toxic overdose, but when combined with massive alcohol ingestion and subsequent withdrawal, this can lead to cerebral excitation propagating to seizure, as both alcohol withdrawal and delta-9-Tetrahydrocannabinol can lead to seizure. Cardiac arrest in such a situation is always dangerous and has serious consequences and even can lead to fatality as known for and cardiac arrest. Timely targeted resuscitative effort can result in a successful outcome in the otherwise young healthy individuals and we should always make a positive resuscitative effort in such cases instead of losing hope. Afterward psychiatric counseling, occupational and behavioral therapies, along with lifestyle modifications can help these people to live longer.

\section{Conclusion}

Cannabis abuse can also lead to serious cardiac dysrhythmia complicated with seizure and ultimately cardiac arrest. If timely and proper resuscitative efforts are instituted, these patients can have a successful outcome.

\section{Table 1. Laboratory Parameters of Patients}

\begin{tabular}{|c|c|c|c|c|}
\hline \multirow{2}{*}{ Parameters } & \multicolumn{2}{|c|}{ Case 1} & \multicolumn{2}{|c|}{ Case 2} \\
\hline & On Admission & On Discharge & On Admission & On Discharge \\
\hline Serum Sodium (135-145 mmol/L) & 144 & 144 & 134 & 141 \\
\hline Serum Potassium (3.5-5 mmol/L) & 3.14 & 3.9 & 3.4 & 4.2 \\
\hline Serum Chloride (98-107 mmol/L) & 108 & 112 & 103 & 113 \\
\hline Serum Magnesium (0.62-1.03 mmol/L) & 0.74 & 0.78 & 1.07 & 0.93 \\
\hline Corrected Calcium (2.10-2.55 mmol/L) & 2.20 & 2.38 & 2.29 & 2.22 \\
\hline Blood Urea (2.5-6.7 mmol/L) & 3.1 & 3.7 & 2.4 & 1.3 \\
\hline Serum Creatinine (50-98 $\mu \mathrm{mol} / \mathrm{L})$ & 79 & 57 & 84 & 57 \\
\hline Serum Protein (60-83 g/L) & 73 & 48 & 67 & 47 \\
\hline Albumin (35-50 g/L) & 43 & 27 & 39 & 27 \\
\hline Total Bilirubin $(<21 \mu \mathrm{mol} / \mathrm{L})$ & 14 & 23 & 8 & 3 \\
\hline Alkaline Phosphatase (40-150 IU/L) & 68 & 75 & 92 & 67 \\
\hline ALT $(<55 \mu \mathrm{mol} / \mathrm{L})$ & 29 & 28 & 74 & 31 \\
\hline GGT $(<36 \mu \mathrm{mol} / \mathrm{L})$ & 33 & 86 & 51 & 24 \\
\hline Creatinine Kinase $(<200 \mu \mathrm{mol} / \mathrm{L})$ & 143 & 1162 & 483 & 311 \\
\hline Troponine $(<0.05$ ng/mL) & Negative & N/A & Negative & $\mathrm{N} / \mathrm{A}$ \\
\hline CRP (0-1 mg/L) & 2.3 & 143 & 0.4 & 52.2 \\
\hline pH (7.35-7.45) & 7.205 & N/A & 7.35 & N/A \\
\hline $\mathrm{PaO}_{2}(10.67-13.33 \mathrm{kPa})$ & 87.10 & N/A & 34.4 & N/A \\
\hline $\mathrm{PaCO}_{2}(4.67-6.0 \mathrm{kPa})$ & 5.28 & N/A & 3.07 & N/A \\
\hline $\mathrm{HCO}_{3}^{-}(23-28 \mathrm{mmol} / \mathrm{L})$ & 15.2 & N/A & 15.8 & N/A \\
\hline $\mathrm{BE}(-2.5$ to +2.5$)$ & -9.8 & N/A & -10.8 & $\mathrm{~N} / \mathrm{A}$ \\
\hline $\mathrm{SaO}_{2}(75-99 \%)$ & 99.6 & N/A & 98.5 & N/A \\
\hline $\mathrm{COHb}(0.5-2.5 \%)$ & 2.2 & N/A & 2.4 & N/A \\
\hline Glucose (3.3-6.1 mmol/L) & 10.6 & N/A & 8.4 & N/A \\
\hline Serum Lactate (0.4-2.2 mmol/L) & 11.2 & N/A & 10.2 & N/A \\
\hline Haemoglobin (11.5-17.4 g/dL) & 14.5 & 9.5 & 13.8 & 10.7 \\
\hline White Cell Count (4.0-10×109/L) & 10.9 & 11.3 & 9.2 & 4.9 \\
\hline Platelets (140-400×109/L) & 392 & 176 & 383 & 292 \\
\hline PT (13-16 seconds) & 15.5 & 16.2 & 15.1 & 14.4 \\
\hline INR & 1.2 & 1.2 & 1.2 & 1.1 \\
\hline
\end{tabular}

$\mathrm{ALT}$, alanine aminotransferase; $\mathrm{BE}$, base excess; $\mathrm{COHb}$, carboxyhemoglobin; $\mathrm{CRP}, \mathrm{C}$-reactive protein; $\mathrm{GGT}$, gamma-glutamyl transferase; $\mathrm{HCO}_{3}^{-}$, bicarbonate ion; INR, international normalized ratio; N/A, not available; $\mathrm{PaCO}_{2}$, partial pressure of carbon dioxide, arterial; $\mathrm{PaO}_{2}$, partial pressure of oxygen, arterial; $\mathrm{PT}$, prothrombin time; $\mathrm{SaO}{ }_{2}$, oxygen saturation. 


\section{CASE REPORT}

\section{Article Information}

*Correspondence: Pradipta Bhakta, MD, DNB, MNAMS, FCAI, EDRA Department of Anaesthesia and Intensive Care, University Hospital Limerick, St Nessan's Rd, Dooradoyle, Co. Limerick, V94 F858, Ireland. E-mail: bhaktadr@hotmail.com

Received: Jul. 24, 2018; Accepted: Aug. 15, 2018; Published: Sep. 22, 2018

DOI: 10.24983/scitemed.acri.2018.00082

Copyright $\odot 2018$ The Author(s). This is an open-access article distributed under the terms of the Creative Commons Attribution 4.0 International License (CC-BY).

\section{Funding: None}

\section{Conflict of Interest: None}

Informed Consent: The patients' consents were obtained.

\section{Keywords}

Alcohol withdrawal; cannabis overdose; cardiac arrest; seizure.

\section{References}

1. Charbonney E, Sztajzel JM, Poletti PA, Rutschmann O. Paroxysmal atrial fibrillation after recreational marijuana smoking: another "holiday heart"? Swiss Med Wkly 2005;135(27-28):412-414.

2. Mittleman MA, Lewis RA, Maclure M, Sherwood JB, Muller JE. Triggering myocardial infarction by marijuana. Circulation 2001; 103(23):2805-2809.

3. Gordon E, Devinsky O. Alcohol and marijuana: effects on epilepsy and use by patients with epilepsy. Epilepsia 2001;42(10):1266-1272.

4. George A, Figueredo VM. Alcohol and arrhythmias: a comprehensive review. J Cardiovasc Med (Hagerstown) 2010;11(4):221-228.

5. Rodrigo C, Epa DS, Sriram G, Jayasinghe S. Acute coronary ischemia during alcohol withdrawal: a case report. J Med Case Rep 2011;5:369.

6. McMicken D, Liss JL. Alcohol-related seizures. Emerg Med Clin North Am 2011;29(1):117-124. 